\title{
La filosofia di Biancaneve. Spunti per i tempi che cambiano
}

\author{
di Cristina Muccioli
}

cristinamuccioli1@gmail.com

\begin{abstract}
Nicola Vitale walks a side path to the smooth march of technocratic rationalism. A rationalism dried up in the imagination, in the capacity for vision, in the sense of community belonging that makes us human beings, as Aristotle taught. Snow White, from a Disney fairy tale, returns to its symbolic powerful significance of alchemical narration where everything, even the most divisive evil, finds meaning and place.
\end{abstract}

Keywords: Psychology, Alchemy, Consciousness, Transition, Fairy tale, Myths, Eliade, Freud, Hillman, Grimm, Zambrano

La filosofia di Biancaneve ${ }^{1}$ di Nicola Vitale è un testo neo-sapienziale. Questa filosofia è freschissima e arcaica, per prospettiva e stile di riflessione, quindi sulle prime può disorientare, suscitare obiezioni spontanee.

Si tratta di rendersi disponibili a un cambio radicale di sguardo cui il pensiero critico sull'evoluzione storica ci ha abituati, e si tratta anche di non scegliere dicotomicamente tra i due.

Come avessimo due occhi con funzioni percettive distinte che adoperiamo uno alla volta riducendo l'altro alla cecità, abbiamo effettivamente rinunciato e marginalizzato l'uso di immagini simboliche per raccontare il reale privilegiando e integrando sempre più massicciamente il rigore scientista delle verità provate, del sapere certamente già saputo e vagliato, già saputo e provato. Pensiero e immaginazione, giudizio critico e fantasia estetica tornano a dialogare rigogliosamente, armonicamente.

\footnotetext{
${ }^{1}$ N. Vitale, La filosofia di Biancaneve. Spunti per i tempi che cambiano, Moretti\&Vitali, Bergamo 2021.
} 
L'impressione perdurante è che l'autore miri a una rivitalizzazione della psiche, dopo una lettura del suo stato preoccupante ma non disperante. Invischiata nellinerzia furiosa del produrre e del consumare, la coscienza si opacizza, si infiacchisce, resta senza lessico e senza racconto, proprio in epoca in cui furoreggia lo storytelling.

Tutto è fulcrato intorno all'appetito di coscienza, di liberazione della nozione di psiche dalla mappatura onnivora delle neuroscienze che ne hanno fatto esito quantificabile dell'attività elettrica dell'encefalo, della sua corteccia, dei suoi neuroni. A questo inaridimento riduzionistico, per cui la più eterea delle nostre incommensurabili dimensioni viene assegnata a un luogo concreto e corporeo, quindi a un tempo fisico con la sua decadenza, l'autore propone una contromisura speculativa che non vuole misurare, non vuole allocare ma espandere e desomatizzare.

Nicola Vitale è un mitografo contemporaneo. I miti, per Vitale, sono potenti raffigurazioni simboliche, immagini-guida che la cultura produce senza curarsi della convivenza ibridata, ben separata da tutte le matrici logiche con i loro rigorosi apparati discorsivi, dall'epistemologia tassonomica Novecentesca, di conscio e inconscio, nemmeno quando collettivi. Per dare immagine, significato e direzione al nostro vivere storico, l'autore osserva la realtà dal punto di vista inconscio, irrazionale, e razionalmente inscrive, colloca, configura.

La società occidentale, sussume Nicola Vitale, è nata quando al mito si è andato sostituendo il concetto. Del mito, però, si avverte ancora incoercibile desiderio, e lo si desume da una produzione culturale di successo patibolare che spazia dalla fantascienza (Guerre Stellari) alle saghe nordiche (Il Signore degli anelli di Tolkien), sino alle leggende metropolitane declinate in popolari serie televisive. Quel che è liquidato da altri come mera 'industria culturale', appendice consumistica del capitalismo, è qui rivisto e interrogato come risposta a un bisogno intimo e inalienabile di reagire alle domande abissali che pone il terribile, l'infinto, l'ignoto, ma anche il sublime e tutto il vitalismo 
infantile che rischia altrimenti di inaridire e svaporare. Va altresì detto che continuiamo, perlopiù inavvertitamente e inconsapevolmente, a parlare la lingua del mito ogni volta che pronunciamo la parola "eco" o che alludiamo al "narcisista" o al "narcisismo", sottendendo la narrazione mitica di Ovidio, con la sua insuperata intuizione sulla specularità e corrispondenza che di per sé non garantiscono affatto comunicazione, né relazione, né quindi amore.

La prima di queste immagini è quella tribale.

Si chiede al lettore di immaginare una piccola tribù e una capanna, destinata poi ad aumentare in dimensioni e ad essere sovraffollata, ingestibile nella contesa di spazi e dinamiche. Ł̀ così che ha inizio la diaspora frammentante, la proliferazione di gruppi, traiettorie esplorative e residenziali, lingue usi e costumi.

Si tratta di una sintesi per immagine, che in sé include anche la grotta, nostro primo riparo da genere Homo, solo ultimamente sapiens. La paleoantropologia e l'archeologia non raccontano una storia diversa, usano termini e metodi diversi, scarto, ma si sa che nel mito lo scollamento dalla realtà non è mai totale. Eravamo cacciatori raccoglitori, e lo siamo stati a lungo. Per il 99,5\%, assicura Giorgio Manzi ne L'ultimo Neanderthal racconta. Storie prima della storia ${ }^{2}$, la nostra storia si è svolta nel Paleolitico. Prima che cacciatori-raccoglitori, due milioni e cinquecentomila anni fa, siamo stati scavengers, saprofagi, essendo ancora incapaci di cacciare e sprovvisti di armi biologiche che ci rendessero atti alla competizione con altri predatori. Anche partendo da una distanza molto più ravvicinata, dalla comparsa di sapiens che data duecentomila anni, torniamo al vagabondare in cerca di selvaggina e frutti spontanei, in grotte e capanne, in protezioni precarie che solo con la scoperta dell'agricoltura, circa novemila anni fa, sono diventate abitazioni, dal latino habeo, avere, stabilmente possedere per stare e per restare.

${ }^{2}$ G. Manzi, L’ultimo Neanderthal racconta. Storie prima della storia, Il Mulino, Bologna 2021. 
Perché parlare di paleoeventi, ci si chiederà. Vitale non lo dice, e si tenta di interpretare la sua evocazione della tribù primordiale, della capanna. La scrittura nasce duemilacinquecento anni fa, quando ancora Socrate era figura mediana, soglia recalcitrante - con ottime ragioni - tra la civiltà orale e quella che tecnicamente chiamiamo storia, perché basata su reperimento di fonti scritte. Anche prima della scrittura, che ha riportato e trascritto quanto ci siamo sempre detti, narrati, tramandato, i saperi intorno alle pratiche, così come le innumeri narrazioni cosmogoniche e mitologiche, sono state vive, capaci di amalgamare comunità e appartenenze, di configurare senso e indirizzo etico.

I filosofi sono tradizionalmente considerati maestri nel rendere complicate le cose semplici, eppure sono tra coloro che azzardano con grande audacia - a partire da Platone - a proporre nelle loro argomentazioni i miti. I miti fanno apparire semplice quel che è complesso, inestricabile groviglio teorico e pratico di problemi. Paradossalmente portano, allo stesso tempo, a problematizzare e a riflettere su concetti che parrebbero eminentemente intuitivi.

I miti sono un patrimonio, non solo per noi occidentali, sono l'individuazione di un pattern ricorrente e strutturale per non smarrirsi atomizzati nel turbinio caotico degli eventi. Se tenuti insieme da un filo interpretante, anche quelli negativi acquistano un senso, si fanno sopportabili, anzi si fanno innesco di migliorie e rammendi esistenziali.

Nel paradigma mitico della rinascita, per esempio, che presuppone la caduta, la morte, Vitale inscrive la diaspora dei dissidenti insofferenti alla costrizione turbolenta della capanna. Quelli della tribù che se ne sono andati hanno patito una mortificazione personale, perché non si può vivere davvero senza appartenere ma solo sopravvivere; e hanno inferto un patimento sociale all'integrità della tribù, che conosce la mancanza, la lacerazione, lo strappo identitario. Nostalgia e perdita, passioni tristi potremmo dire, si fanno però causa dell'effetto ritorno e ricongiungimento su un piano di più consapevole e 
rispettosa organizzazione. La grande capanna prevederà i monasteri al suo interno, spazi inclusi di esclusione volontaria, separazione integrata nello spazio della relazione.

Vitale non attinge dalla classicità, crea un cortocircuito tra la preistoria della caverna e la modernità di Biancaneve, fiaba popolare europea scritta dai fratelli Grimm nel 1819, che certamente avranno attinto a repertori più antichi nel riformulare la vicenda che acquisterà fama planetaria con Walt Disney, pure citato per la questione dello spechio d'acqua in cui la principessina vede riflesso il volto di colui che la salverà dal sonno eterno $\mathrm{e}$ maledetto da una pozione velenosa.

Biancaneve compare solo alla fine del saggio, negli ultimi paragrafi dell'epilogo. Lì il saggio mostra il suo scheletro trasparente, la sua ispirazione e il suo indirizzo tutto agilmente bilanciato tra momenti di morte (apparente) del vitalismo culturale e spirituale e la sua rinascita (più autentica che apparente). Fedele e anche appassionato al tema della trasmutazione, cuore pulsante della sapienza e dell'arte alchemica, Vitale si muove con atletica destrezza tra accostamenti tematici e gli autori più disparati, con molta coerenza e ordine nella varietà.

Da Cicerone a Eliade, da Kant all'immancabile Heidegger, da Vico a Marx, da Freud a Jung a Hillman, l'autore non evoca evocare una donna, una pensatrice, una poetessa, una filosofa. Un rilievo questo che parrà trascurabile e gratuito, ma che nasce senza livore dalle aspettative suscitate dal titolo stesso del saggio. L'unica presenza femminile, infatti, è Biancaneve, una figura fiabesca nata - con il conforto di una pluricentenaria tradizione interpretativa - dal sapere e dall'immaginario alchemico, che si auspicava in antitesi o in alternativa all'impianto razionalistico sino a che l'autore non scrive "scienza alchemica".

Lo schema archetipico che fa di Biancaneve un emblema alchemico comincia dalle apparenze, dai colori che già il nome in parte contiene: sono il bianco niveo della sua carnagione (albedo, il colore lunare della purezza 
sorgiva che riesce ad accendere il buio opaco della notte), il nero dei capelli (nigredo, opera al nero, il primo passo del percorso di creazione della pietra filosofale che necessita di decomposizione e morte dell'esistente), il rosso acceso delle guance (corrispondente alla rubedo il calore, la vita, il magma emozionale, il compimento sintetico e armonico dei conflitti).

Da fatterello trascurabile, da comparsa vicina all'insignificanza, tutto nel mito alchemico si fa segno, simbolo, metafora, tutto concorre a dare immagine e voce narrante all'avvicendarsi di periodi cupi, sofferenti a quelli di risveglio e rinvigorimento. I sette nani, per esempio, vanno alla ricerca dell'occultus lapis nelle viscere della terra, del corpo-inconscio, protagonisti di un viaggio ermetico al centro oscuro e insidioso che custodisce ogni vera preziosità luminosa che per rilucere necessità lavoro, immersione, sgrezzamento del piombo in oro.

Il bosco intricato e oscuro, minaccioso e pauroso in cui la fanciulla perseguitata dalla matrigna malvagia e invidiosa è costretta a cercare riparo, in fuga, è topos letterario tra i più sedimentati nell'immaginario di tutti a partire da Dante. Da foresta, ad aggiunta innecessaria, viene "forestiero", e tutto quello che l'alterità per eccellenza a ciò che esce dai confini della convivenza civile porta con sé. Occorre attraversarla, però, secondo la rilettura di Vitale, non semplicemente schivarla. Solo andando a tentoni, a mani nude tra l'impervio, si raffineranno le abilità e la forza autentica per uscirne rinvigoriti, forti di amici e alleanze che nemmeno sapevamo di avere. Proprio nel fitto della boscaglia si aprono piccoli, salvifici spiazzi erbosi allagati di luce, di opportunità, quindi di potenza.

Viene in mente Maria Zambrano, leggendo questo saggio, con il suo Chiari di bosco: «E la vista lontana dal centro appena visibile, e la vista che i chiari del bosco offrono, sembrano promettere, più che una visione nuova, un mezzo di visibilità in cui l'immagine sia reale e il pensiero e il sentire si identifichino senza che ciò sia a costo di perdersi l'uno nell'altro o di annullarsi. Una visibilità nuova, luogo di conoscenza e di vita senza distinzione, pare che sia 
il magnete che ha orientato tutto questo percorso analogamente a un metodo di pensiero. Ogni metodo salta fuori come un "Incipit vita nova" che tende verso di noi con la sua inalienabile allegria» ${ }^{3}$.

\section{Nota bibliografica}

MANZI, Giorgio, L’ultimo Neanderthal racconta. Storie prima della storia, Il Mulino, Bologna 2021.

VITALE, Nicola, La filosofia di Biancaneve. Spunti per i tempi che cambiano. Moretti\&Vitali, Bergamo 2021.

ZAMBRANO, Maria, Chiari di bosco, Bruno Mondadori, Milano 1977.

${ }^{3}$ M. Zambrano, Chiari di bosco, Bruno Mondadori, Milano 1977, p. 14. 